\title{
RETRACTED ARTICLE: Stability analysis of dynamic collaboration model under two-lane case
}

\author{
Zhipeng Li · Run Zhang • Shangzhi Xu • \\ Yeqing Qian
}

Received: 8 October 2013 / Accepted: 1 March 2014 / Published online: 26 March 2014

(C) Springer Science+Business Media Dordrecht 2014

This article has been retracted by the Editor-in-Chief of Nonlinear Dynamics. The authors admitted that two figures and several paragraphs of text (in particular: Figs. 2 and 3, the first and last paragraphs in section 2.1, and all of section 3, except the final sentence.) included had been reproduced without credit or citation from "Influence of lane change on stability analysis for two-lane traffic flow", by Zheng Liang, Ma Shou-Feng and Zhong Shi-Quan, in Chinese Journal of Physics, B Volume 20, Number 8, (2011).

Z. Li · R. Zhang $(\varangle) \cdot$ S. Xu · Y. Qian The Key Laboratory of Embedded System and Service Computing Supported by Ministry of Education, Tongji University, Shanghai 201804, China e-mail: 12zhangrun@tongji.edu.cn

Z. $\mathrm{Li}$

e-mail: lizhipeng@tongji.edu.cn 